\title{
Antenna array synthesis using a newly evolved optimization approach: Strawberry algorithm
}

\author{
Konidala R. Subhashini*
}

\begin{abstract}
An attempt has been made for the first time to apply this proposed Strawberry optimization technique to antenna array synthesis problem. The case study cited here refer to linear and circular array configurations. The design constraints are limited to minimizing the side lobe level and restricting the first null beam width, which play significant roles in antenna array performances. The key parameters which greatly influence in achieving the said objectives are either placement of antenna elements or amplitudes of excitations of these elements or both. And the recently reported meta heuristic nature inspired optimization algorithms have addressed to these problems quite effectively and the exciting result obtained using the said approach has undoubtedly proved the strawberry algorithm as a potential contender in the optimization domain.

K e y w or d s: strawberry algorithm, antenna array, optimization, peak SLL, null depth
\end{abstract}

\section{Introduction}

This piece of research work is primarily aimed at establishing the superiority of the proposed optimization algorithm and conscious effort is made to validate the claim in a systematic matter by applying the same in antenna array synthesis problem. Recently, nature inspired meta heuristic optimization techniques have gained significant importance in solving non-linear problems quite successfully in comparison to their conventional counterparts based on the error derivative techniques which often suffer from trapping in local minima. The encouraging results achieved by applying of such evolutionary methods have motivated the present authors to pick one of the most recently reported meta heuristic approach known as Strawberry Algorithm[SBA] [1] and to study how effective it is in contributing to the performance gain by optimizing the parameters (positions and excitations) of antenna elements. The strawberry algorithm as an optimization tool has been applied for the first time to antenna array synthesis and amazingly faster convergence characteristic is obtained in addition to remarkable improvement in side-lobe suppression and prescribed null placements. However, there have been a continuous and substantial growth in development of many efficient stochastic optimization techniques which have been successfully applied to antenna array problems with promising results and to name a few are GA [2], DE [3], ACO [4], PSO [2], CSO [5], IWO [6], ALO [7,8], SMO [9], etc. The Strawberry algorithm has tasted success rate in other fields such as automation [10], micro/smart grid problems [11]. The current focus is to explore the possibility of identifying Strawberry algorithm as a successor in finding optimal solutions in nonlinear problems, with particular emphasis to antenna array synthesis. And in fact the results obtained have clearly paved the way for the proposed algorithm.

\section{An overview of strawberry algorithm [1]}

The essential components embedded within this nature inspired algorithm are global search as well as local search abilities. These combined strategies have evolved a powerful optimization concept where the chances of the solution getting stuck at local minima are extremely bleak. Both the exploration and exploitation in the search space of finding an optimum solution are achieved using global and local searches respectively. The philosophy in relation to strawberry plant taking part in optimization procedure lies with this fact that it comprises both runners and roots, the two most powerful components which decide the course of action leading to convergence with optimal solution. While the runners play a significant role in global search as they have a greater reach, the roots dedicate themselves in local searches because they tend to move in small steps around a given global domain to find for the solution. And the process of finding the objective continues with each iteration with every mother plant giving birth to a daughter plant carrying the same characteristics to maintain the legacy. The runners and roots belonging to a plant act as active agents to accomplish their mission and in doing so the mother plants sacrifice their lives for the survival of the daughter plants, which form the fundamental principle of evolution. This ordeal, which is a perpetual struggle to find food for the very existence of the plant mimics a process of optimization.

*Department of Electrical Engineering, National Institute of Technology Rourkela, India, subhashiniratna@gmail.com 


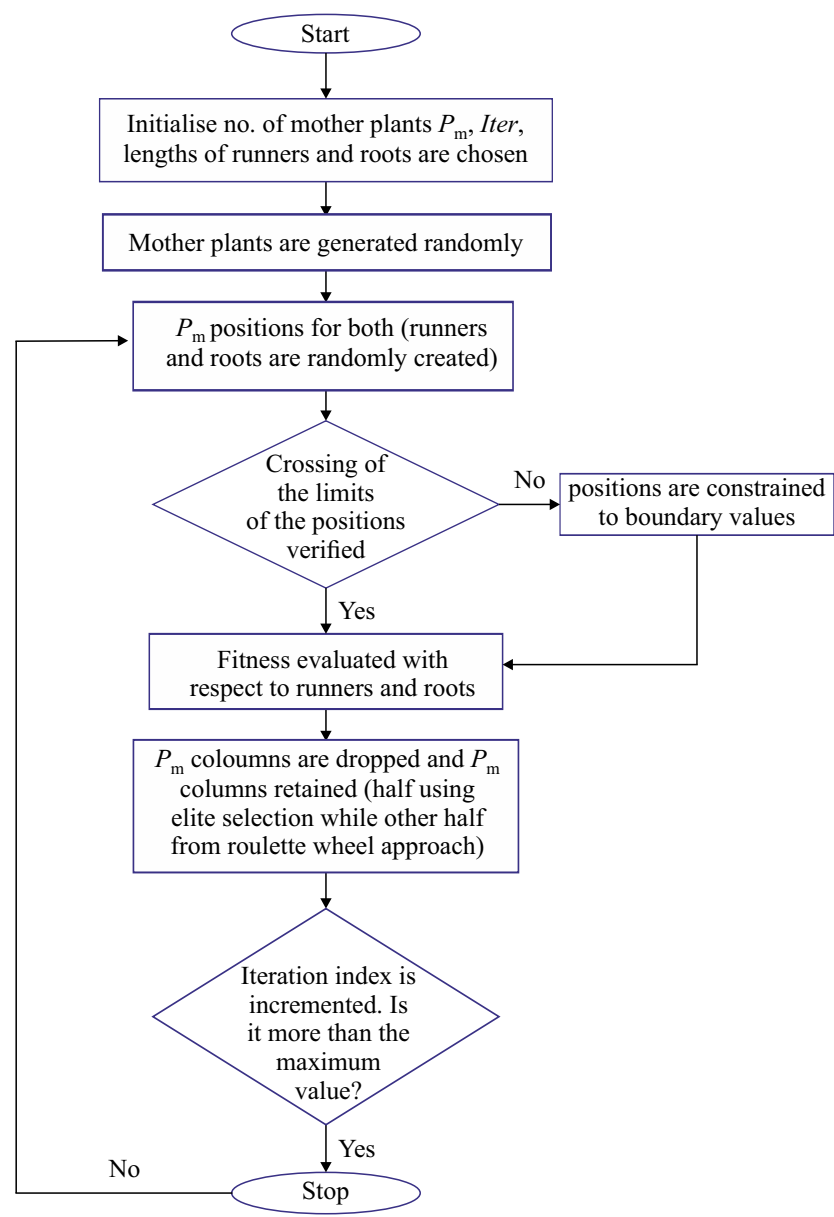

Fig. 1. Flowchart of the conventional SBA

The accompanied flowchart in Fig. 1 depicts this process of optimization.

\section{Antenna array analysis}

\subsection{Linear antenna array $(L A A)$}

In a linear Antenna Array (LAA), Fig. 2, a configuration comprising of $M=2 Q$ elements located along the $x$-axis, the corresponding array factor is governed by the relation $[12]$

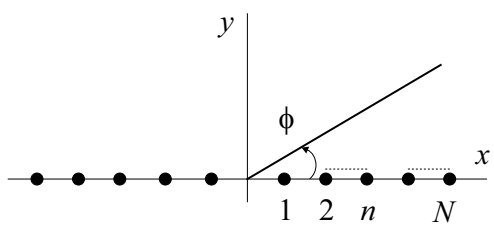

Fig. 2. Geometry of $2 N$ element symmetric linear array placed along the $x$-axis

$$
A F_{\text {lin }}(\theta)=2 \sum_{r=1}^{Q} I_{r} \cos \left(c x_{r} \cos \theta\right)
$$

where $c$ is the wave number represented by $c=2 \pi / \lambda, \theta$ is the azimuth angle bounded between $0^{\circ}$ to $180^{\circ}$ and $x_{r}$ and $I_{r}$ denote the position and the excitation amplitude of the $r^{\text {th }}$ antenna element respectively and the phase is assumed to be zero. The $2 Q$ elements are symmetrically arranged on each side centered around $y$-axis which forms the total elements of the array and denoted as $M$. In order to achieve the desired objective of minimizing the sidelobe level, the array factor $A F_{\text {lin }}(\theta)$ is optimized using the prescribed strawberry algorithm to determine the best solutions for antenna element locations or their excitation amplitudes as per the criteria.

The objective function chosen for the purpose is given by $[13]$.

$$
F_{1}=\min \left[\max \left(20 \log _{10} \frac{\left|A F_{l i n}(\theta)\right|}{\max \left|A F_{l i n}(\theta)\right|}\right)\right] .
$$

While the objective function is minimized to find out antenna element positions with uniform current excitation the following constraints are imposed with reference to inter spacing distances $\left|\left(x_{i}-x_{j}\right)\right|>0.25 \lambda \min \left(x_{i}\right)>$ $0.25 \lambda$, for $i=1,2,3, \ldots, Q, i \neq j$. Similarly, when another restriction of a very wide null is presumed, the objective function is accordingly modified to consider this along with minimizing the SLL beyond the prescribed zone as stated below

$$
F_{2}=\left.\alpha_{1} F_{1}\right|_{\theta \in S_{1}}+\left.\alpha_{2} F_{1}\right|_{\theta \in S_{2}}
$$

where $S_{1}$ and $S_{2}$ are domains of the scanning angle representing the SLL suppression boundary and very wide null or deep null limitations as decided. $\alpha_{1}$ and $\alpha_{2}$ are the coefficients chosen depending upon the objective to be fulfilled.

\subsection{Circular antenna array $(C A A)$}

Arrays with circular arrangement, Fig. 3, has a very practical interest. Literature suggests that various applications of circular antenna arrays in sonar, radar, mobile, and commercial satellite communication systems.

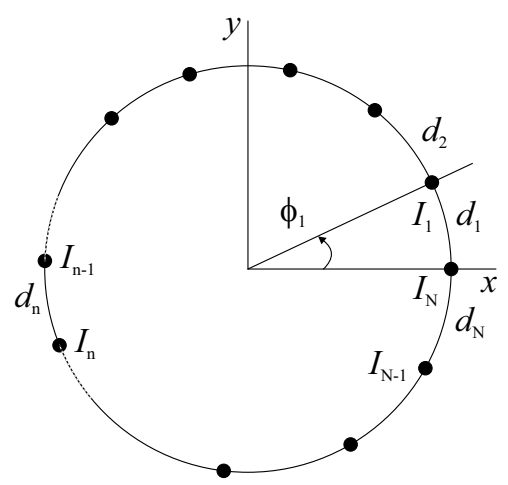

Fig. 3. Circular arrangement of isotropic elements with uniform inter element spacing 
Table 1. Optimized position $2 Q=10$ element symmetrical (LAA) with fixed FNBW

\begin{tabular}{lcccccc}
\hline Optimization Method & \multicolumn{5}{c}{ Optimized element positions } & PeakSLL(dB) \\
\hline SMO [9] & 0.236 & 0.528 & 1.007 & 1.471 & 2.126 & -20.25 \\
ACO [4] & 0.25 & 0.55 & 1.05 & 1.55 & 2.15 & -18.27 \\
PSO [13] & 0.2040 & 0.6278 & 1.6641 & 1.4564 & 2.3033 & -19.71 \\
SBA (proposed) & 0.230 & 0.515 & 0.981 & 1.460 & 2.120 & -22.73 \\
\hline
\end{tabular}
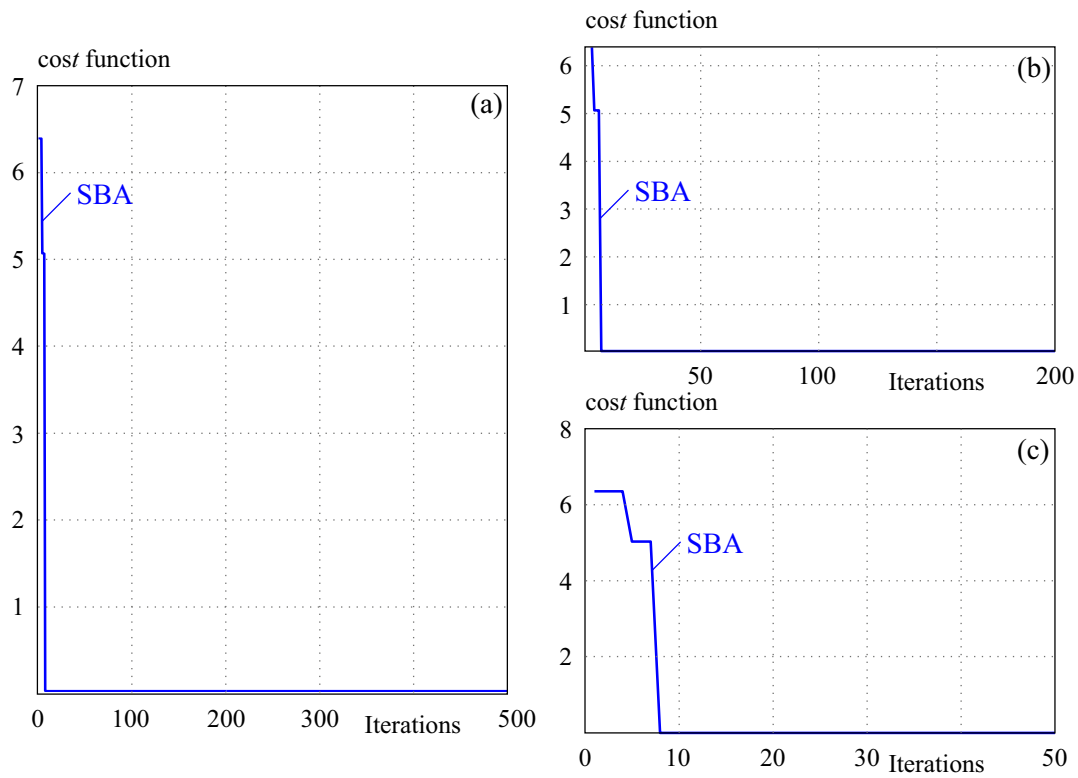

Fig. 4. Convergence characteristic and its magnified views

The radiation pattern which is the performance indicator of the array depends on the number of elements, spacing and amplitude (excitation) distribution. Synthesis of the circular array is done through finding out the optimum parameters as stated above which is further checked for its optimal performance. In order to effectively carry out the optimization the fitness function $F_{3}$ is chosen with the restriction that the aperture size is limited to a particular range which is interconnected to the number of elements [6] and the corresponding array factor and the objective function are

$$
\begin{gathered}
A F_{\text {cir }}(\phi)=\sum_{r=1}^{Q} I_{r} e^{j\left(k a \cos \left(\phi-\phi_{r}\right)\right)} . \\
F_{3}=c_{1} F_{3}^{N U L L}+c_{2} F_{3}^{S L A}+c_{3} F_{3}^{M S L} \\
F_{3}^{N U L L}=\left|A F_{\text {cir }}\left(\phi_{\text {null } 1}\right)\right|+\left|A F_{\text {cir }}\left(\phi_{\text {null } 2}\right)\right|, \\
F_{3}^{S L A}=\frac{1}{\pi+\phi_{\text {null } 1}} \int_{-\pi}^{\phi_{\text {null1 } 1}}\left|A F_{\text {cir }}(\phi)\right| \mathrm{d} \phi \\
+\frac{1}{\pi-\phi_{\text {null2 }}} \int_{\phi_{\text {null } 2}}^{\pi}\left|A F_{\text {cir }}(\phi)\right| \mathrm{d} \phi
\end{gathered}
$$

$$
F_{3}^{M S L}=\left|A F_{c i r}\left(\phi_{M S L 1}\right)\right|+\left|A F_{c i r}\left(\phi_{M S L 2}\right)\right| .
$$

Further, the lower and upper nulls(angles) referring to the major lobe $\phi_{\text {null1 } 1}$ and $\phi_{\text {null } 2}$ respectively. The process of optimization takes into cognizant the maximum side lobe spread in the in the lower azimuth range and upper azimuth range denoted by $\phi_{M S L 1}$ and $\phi_{M S L 2}$ respectively. The angles in the lower span are from $(-\pi$ to $\left.\phi_{\text {null1 } 1}\right)$ and that in upper span extends from $\left(\phi_{\text {null } 2}\right.$ to $\pi$ ) respectively. The values $c_{1}, c_{2}$ and $c_{3}$ are weighting/scaling factors. Each term embedded in the objective function is biased and influenced by their corresponding scaling factor.

Here $Q$ represents the total number of elements in the circular array. $I_{r}$ and $\phi_{r}$ are the excitation amplitude and angular position of the $r^{\text {th }}$ element respectively, $c$ is the wave number given by $2 p i / \lambda$ and $a$ is the radius of the circular arrangement. Alternatively $c a$ and $\phi_{r}$ can be described as

$$
c a=2 \pi a / \lambda=\sum_{i=1}^{Q} d_{i},
$$

$\phi_{r}=2 \pi / c a \sum_{i=1}^{a} d_{i}$, where $d_{i}$ is the arc length between the $i^{\text {th }}$ and $(i-1)^{\text {th }}$ elements. 


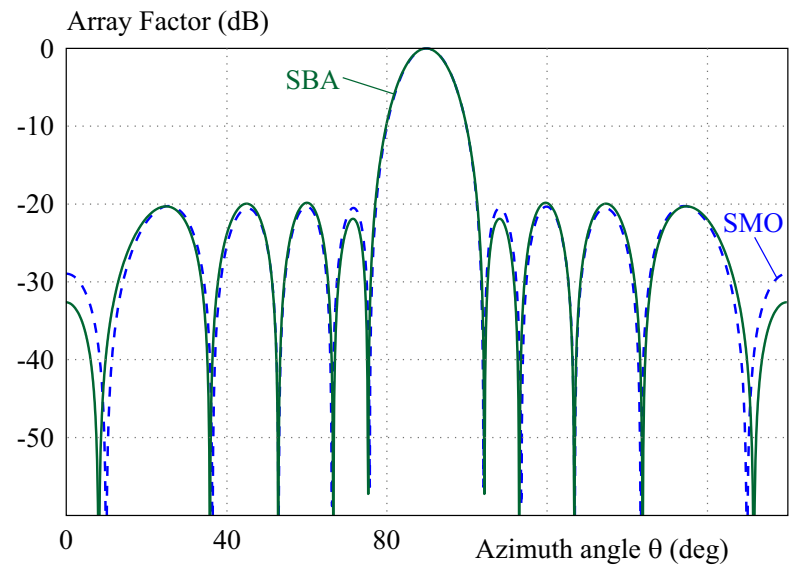

Fig. 5. Array pattern of 10 eloements LAA with constant FNBW

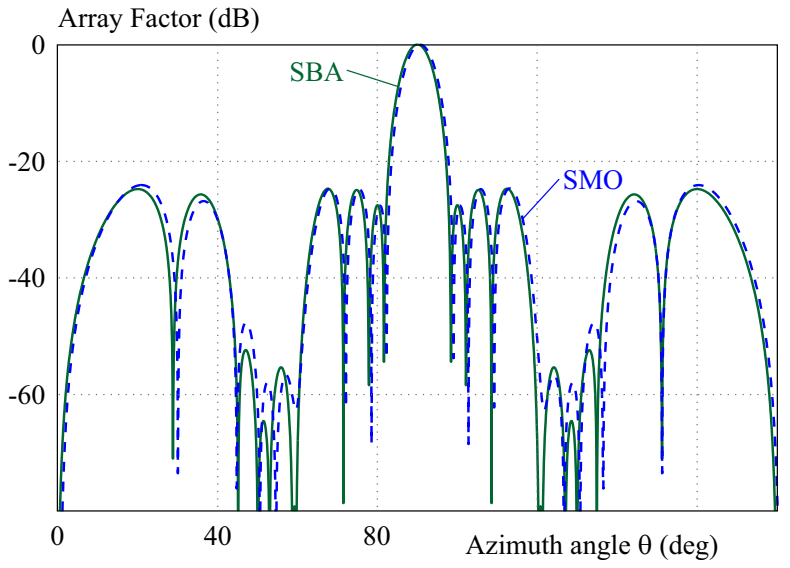

Fig. 6. Array pattern for $M=10$ elements (LAA) with wide null1

Table 2. Optimized excitation amplitudes of 10 elements (LAA) for very wide null

\begin{tabular}{lccccccccccc}
\hline Optimization method & \multicolumn{10}{c}{ Optimized current amplitudes } \\
\hline SMO [9] & 0.772 & 0.771 & 0.773 & 0.645 & 0.496 & 0.505 & 0.368 & 0.461 & 0.199 & 0.166 \\
SBA (Proposed) & 0.768 & 0.752 & 0.777 & 0.629 & 0.500 & 0.498 & 0.362 & 0.455 & 0.198 & 0.163 \\
\hline
\end{tabular}

Table 3. Optimized position of $2 Q=32$ elements of (LAA) with single null

\begin{tabular}{|c|c|c|c|c|c|c|c|c|c|c|}
\hline \multirow{2}{*}{$\begin{array}{l}\text { Optimization method } \\
\text { depth }(\mathrm{db}) \\
\text { SBA (Proposed) }\end{array}$} & \multirow{3}{*}{$\begin{array}{l}0.2305 \\
0.5029\end{array}$} & \multicolumn{7}{|c|}{ Optimized current amplitudes } & \multirow{2}{*}{$\begin{array}{c}\text { Peak } \\
-20.80\end{array}$} & \multirow{2}{*}{$\begin{array}{c}\text { Null } \\
\text { SLL }(\mathrm{dB}) \\
-93.49\end{array}$} \\
\hline & & 0.8476 & 1.5532 & 2.3411 & 3.1724 & 4.1019 & 5.0681 & 6.3408 & & \\
\hline & & 1.1981 & 1.9374 & 2.7519 & 3.6280 & 4.5768 & 5.6885 & 7.0268 & & \\
\hline \multirow[t]{2}{*}{$\mathrm{CSO}[5]$} & 0.2883 & 1.1929 & 1.9768 & 2.6886 & 3.4848 & 4.3822 & 5.4817 & 7.0412 & -18.24 & -80 \\
\hline & 0.6830 & 1.5199 & 2.3247 & 3.1362 & 3.9538 & 4.9252 & 6.2091 & & & \\
\hline \multirow[t]{2}{*}{ ALO [7] } & 0.2121 & 1.2499 & 2.2355 & 3.0146 & 3.7627 & 4.7500 & 5.7500 & 7.2500 & -15.5 & -83.65 \\
\hline & 0.7412 & 1.7500 & 2.6938 & 3.4513 & 4.2684 & 5.2532 & 6.4509 & 7.9990 & & \\
\hline
\end{tabular}

\section{Simulation of case studies}

The antenna array synthesis problem is considered for both linear and circular array configurations in order to validate the worthiness of the proposed method. The sole intention in such studies is to compare the results with other nature inspired meta heuristic algorithms so that inference can be made to accept it as a worthy alternative. The examples were chosen to compare optimized antenna element positions and excitation amplitudes with other reported algorithms subject to the constraint that the most important performance index i.e. side lobe level is minimized while specifying first null beam width (FNBW) within prescribed limits. And such daunting challenges have been adequately taken care of by the proposed algorithm. In order to substantiate a capability of the proposed SBA optimization technique to antenna synthesis problem, the convergence characteristic is plotted in Fig. 4 along with its blown up views IS depicted. This figure refers to design of 10 elements lin- ear array configuration for optimum locations with constrained FNBW. Figure 4 shows a glaring piece of evidence that the convergence characteristic is extremely fast, far exceeding the expectation and around 10 iterations the convergence is settling down.

\subsection{Discussion on results of linear antenna array}

In the case of the linear array three different situations are considered. At first the optimized positions of the antenna elements are determined taking into account FNBW with each element excited with unit amplitude. In the second example a wide null is considered and solution is sought in determining the optimized excitation amplitudes of the antenna elements a uniform spaced arrangements. Similarly, another example with deep null is examined.

\section{EXAMPLE 1 (10 elements).}

In this case study the optimization of position(inter element spacing) of the elements is carried out by min- 


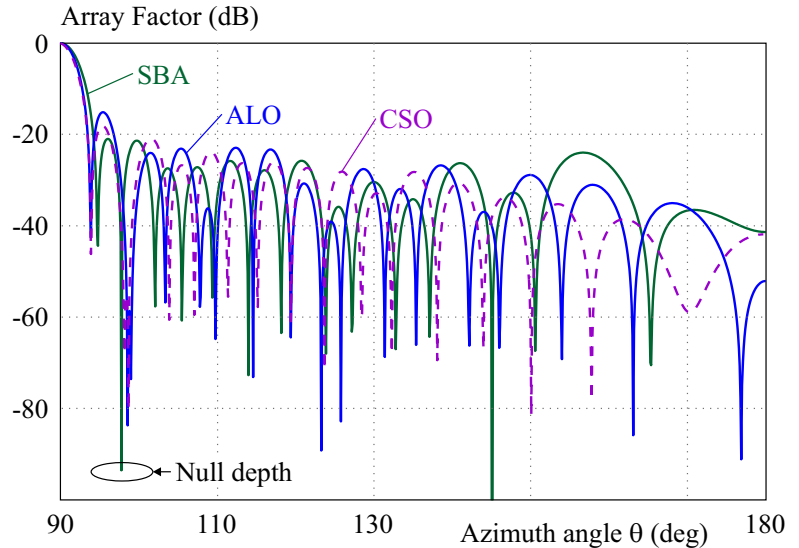

Fig. 7. Array pattern of 32 eloements (LAA) with single null

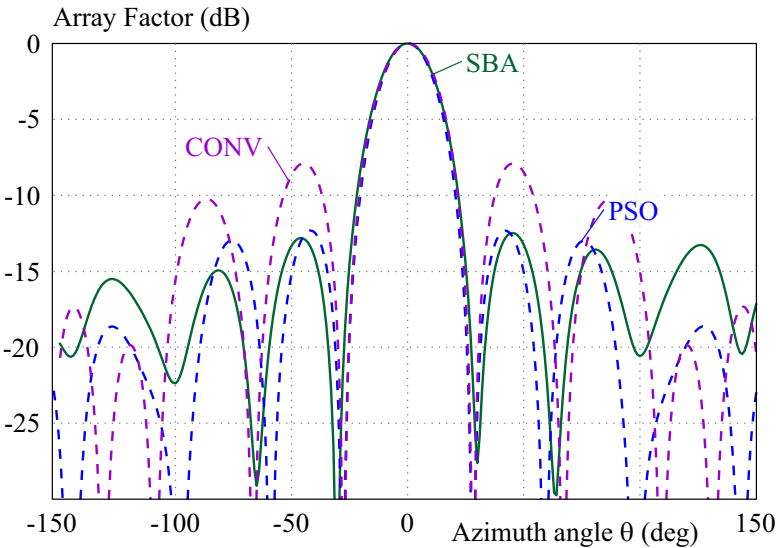

Fig. 8. Array pattern for $M=10$ elements (CAA)

Table 4. Parametric data

\begin{tabular}{lcccc}
\hline Algorithm & population size & Iterations & Independent runs & Function evaluation \\
\hline SBA (Proposed) & 10 & 500 & 5 & 25000 \\
CSO [5] & 50 & 1000 & 15 & 750000 \\
ALO [7] & 40 & 1000 & 15 & 600000 \\
\hline
\end{tabular}

Table 5. Optimized excitation amplitudes and Distances of 10 elements (CAA)

\begin{tabular}{lllllllllll}
\hline Algorithm & \multicolumn{10}{c}{ SBA (Proposed) } \\
\hline Excitations & 0.9942 & 0.7652 & 0.5771 & 0.9561 & 0.7015 & 1.0000 & 0.5281 & 0.7994 & 0.9791 & 0.9792 \\
Distances & 0.3021 & 0.9678 & 0.4206 & 0.9449 & 0.3068 & 0.3508 & 0.9389 & 0.4042 & 0.9536 & 0.3299 \\
\hline Algorithm & & \multicolumn{10}{c}{ PSO [13] } \\
\hline Excitations & 1.0000 & 0.7529 & 0.7519 & 1.0000 & 0.5062 & 1.0000 & 0.7501 & 0.7524 & 1.0000 & 0.5067 \\
Distances & 0.3170 & 0.9654 & 0.3859 & 0.9654 & 0.3185 & 0.3164 & 0.9657 & 0.3862 & 0.9650 & 0.3174 \\
\hline
\end{tabular}

imizing the SLL in the regions $\theta=\left(0^{\circ}\right.$ to $\left.74^{\circ}\right)$ and $\theta=\left(106^{\circ}\right.$ to $\left.180^{\circ}\right)$. The strawberry algorithm is used to determine the optimized element locations $x_{r}$ with a FNBW condition imposed on it. The array pattern is illustrated in Fig. 5 using SBA and other optimization algorithms SMO [9]. The peak SLL obtained corresponding to SBA and other nature-inspired optimization techniques are summarized in Tab. 1. There is a significant reduction in SLL using the proposed approach as compared to other optimization algorithms such as PSO [13], ACO [4] and SMO [9]. The proposed method (SBA) not only yields a peak SLL of $-22.73 \mathrm{~dB}$ but has an improvement of $-2.48 \mathrm{~dB}$ compared to its nearest competitor SMO [9]. The parameters chosen for the proposed SBA approach are, $P_{m}=10$ and Iter $=10$. Thus to achieve the array pattern as shown in Fig. 3 applying SBA, number of function evaluations is only 100 while that using SMO [9] and PSO [13] are 1200 and 10000 with the reduction in function evaluations of $91 \%$ and $99 \%$ respectively. This clearly represents the impressive achievement in terms of speed of convergence.
EXAMPle 2 (10 elements). This being a complex problem where a very wide null is considered the Iter and $P_{m}$ are selected as 500 and 10 respectively and the two coefficients $\alpha_{1}$ and $\alpha_{2}$ assigned 0.5 each, with number of independent runs being 5 leading to the number of function evaluations to 25000 . The average null depth level using SBA is $-67.47 \mathrm{~dB}$. SMO [9] has given a null depth of $62.39 \mathrm{~dB}$. The radiation pattern is shown in the Fig. 4 and the corresponding magnitudes are given in Table 2 . This is in complete contrast with SMO [9] where for the same array pattern as shown in the Fig. 6 the number of function evaluations are 200000. Further, the proposed approach picks up the optimum result from 5 data sets where as SMO [6] does the same from 20 data sets. It indicates the superiority of the algorithm and its robustness.

EXAmple 3 (32 elements). Another experiment was carried out to prove the consistency of the proposed SBA technique to effectively handle varieties of the problem in antenna design domain. Here a 32 element linear array is considered where the prescribed null is at $\theta=81^{\circ}$ 
(and $\theta=99^{\circ}$, the mirror image with reference to $y$-axis at $90^{\circ}$ taking a symmetrical configuration). In addition to the usual objective remains the same of minimizing the SLL in the entire scanning angle $\left(\theta=0^{\circ}\right.$ to $\left.180^{\circ}\right)$ leaving apart the main lobe $\left(\theta=84^{+} \circ\right.$ to $\left.96^{\circ}\right)$. The performance results have been quite significant in the case of SBA in comparison to CSO [5] and ALO [7]. Table 3 summarizes the optimized locations using different algorithms while the antenna elements are excited uniformly. The array pattern shown in the Fig. 7 is self explanatory and clearly speaks about the highly encouraging results obtained using SBA in terms of remarkable improvement in peak SLL (20.89 dB) and null depth $(93.49 \mathrm{~dB})$ when compared to the previous best contenders ALO [7], winning by a wide margin.

The parametric selection of various algorithms are listed in the Table 4. It is clear that a massive saving of function evaluation to the tune of $96 \%$ and $95.8 \%$ are achieved in comparison to CSO [5] and ALO [7] respectively.

\subsection{Discussion on results of circular antenna array}

In order to encompass the domain of application to a further level, the circular array synthesis is brought into its ambit. Both the optimized element locations and their amplitude have been determined considering the aperture size and side lobe level for an antenna array with $N=10$ elements. The application of the proposed algorithm has witnessed an encouraging result for this case study also and there by consolidates the position that Strawberry algorithm for optimization can definitely contribute in evaluating optimum design results. The performance attributes i.e, the maximum sidelobe level, the beam width and the minimum circumference $(c a)$ obtained by applying the proposed algorithm are $13.29 \mathrm{~dB}, 22.40^{\circ}$ and $5.9197 \lambda$ respectively. The comparison is carried out with PSO [13] to prove the effectiveness of the proposed scheme and are reported to be $12.3 \mathrm{~dB}, 24.34^{\circ}$ and $5.9029 \lambda$. Figure 8 shows the radiation patterns for the circular configuration considered. It is deduced from the numerical analysis given in Table 5 that the proposed SBA has surpass the PSO [13] in terms of beam width and maximum sidelobe level. So far as the parameter selection of SBA are concerned, $P_{m}=10$, Iter $=500$ and 50 independent trials are chosen. The objective function constants $c_{1}, c_{2}$ and $c_{3}$ are fixed as $0.4,0.2$ and 0.4 respectively after carrying out a thorough study, by varying the weighting factors.

\section{Conclusion}

All the computations are performed with MATLAB R2013a on a PC with Intel (R) core (TM) i5 CPU and 4.00 GB RAM. It is absolutely evident from the simulation results that the SBA has performed exceedingly well in terms of achieving extremely fast convergence and significant reduction in number of function evaluations as well as performance indices $i e$ be it side lobe level or null placements in prescribed locations. Further, in comparison with its counterparts in the nature inspired optimization domain, the SBA has in many occasions outclassed them with reference to performance and very rightfully has claimed to occupy a respectable position in the optimization arena.

\section{REFERENCES}

[1] F. Merrikh-Bayat, "A Numerical Optimization Algorithm Inspired by the Strawberry Plant", preprint arXiv:1407.7399, 2014.

[2] M. M. Khodier and C. G. Christodoulou, "Linear Array Geometry Synthesis with Minimum Sidelobe Level and Null Control using Particle Swarm Optimization", IEEE Transactions on Antennas and Propagation vol. 53, no. 8, pp. 2674-2679, 2005.

[3] F. Zhang, W. Jia, and M. Yao, "Linear Aperiodic Array Synthesis using Differential Evolution Algorithm", IEEE Antennas and Wireless Propagation Letters vol. 12, pp. 797-800, 2013.

[4] E. Rajo-Iglesias and O. Quevedo-Teruel, "Linear Array Synthesis using an Ant-Colony-Optimization-Based Algorithm", IEEE Antennas and Propagation Magazine vol. 49, no 2, pp. 70-79, 2007.

[5] L. Pappula and D. Ghosh, "Linear Antenna Array Synthesis using Cat Swarm Optimization", AEU-International Journal of Electronics and Communications vol. 68, no. 6, pp. 540-549, 2014.

[6] G. G. Roy, S. Das, P. Chakraborty, and P. N. Suganthan, "Design of Non-Uniform Circular Antenna Arrays using a Modified Invasive Weed Optimization Algorithm", IEEE Transactions on antennas and propagation vol. 59, no. 1, pp. 110-118, 2011.

[7] P. Saxena and A. Kothari, "Ant Lion Optimization Algorithm to Control Side Lobe Level and Null Depths in Linear Antenna Arrays", AEU-International Journal of Electronics and Communications vol. 70, no. 9, pp. 1339-1349, 2016.

[8] K. R. Subhashini and J. K. Satapathy, "Development of an Enhanced Ant Lion Optimization Algorithm and its Application in Antenna Array Synthesis", Applied Soft Computing vol. 59, pp. 153-173, 2017.

[9] A. A. Al-Azza, A. A. Al-Jodah, and F. J. Harack-iewicz, "Spider Monkey Optimization: A Novel Technique for Antenna Optimization", IEEE Antennas and Wireless Propagation Letters vol. 15, pp. 1016-1019, 2016.

[10] B. S. Charan, A. Mittal, and R. Tiwari, "Multirobot Navigation in Unknown Environment using Strawberry Algorithm", International Journal of Robotics Applications and Technologies (IJRAT) vol. 5, no. 1, pp. 63-81, 2017.

[11] H. N. Khan, H. Iftikhar, S. Asif, R. Maroof, K. Am-breen, and N. Javaid, "Demand Side Management using Strawberry Algorithm and Bacterial Foraging Optimization Algorithm in Smart Grid", in International Conference on Network-Based Information Systems. Springer, 2017, pp. 191-202.

[12] C. A. Balanis, "Antenna Theory: Analysis and Design", John Wiley \& Sons, 2012.

[13] M. M. Khodier and M. Al-Aqeel, "Linear and Circular Array Optimization: A Study using Particle Swarm Intelligence", Progress in Electromagnetics Research B vol. 15, pp. 347-373, 2009.

Received 6 June 2019 